Archives de sciences sociales des religions

$168 \mid 2014$

Bulletin Bibliographique

\title{
Guerre de foi ou guerre sans foi. 1914-1918
}

Frédéric Gugelot

\section{OpenEdition}

Journals

Édition électronique

URL : http://journals.openedition.org/assr/26272

DOI : $10.4000 /$ assr. 26272

ISSN : $1777-5825$

Éditeur

Éditions de l'EHESS

Édition imprimée

Date de publication : 31 décembre 2014

Pagination : 15-28

ISBN : 978-2-7132-2467-6

ISSN : 0335-5985

Référence électronique

Frédéric Gugelot, "Guerre de foi ou guerre sans foi. 1914-1918 », Archives de sciences sociales des religions [En ligne], 168 | 2014, mis en ligne le 30 mars 2018, consulté le 10 décembre 2020. URL http://journals.openedition.org/assr/26272 ; DOI : https://doi.org/10.4000/assr.26272

Ce document a été généré automatiquement le 10 décembre 2020.

(C) Archives de sciences sociales des religions 


\title{
Guerre de foi ou guerre sans foi. 1914-1918
}

\author{
Frédéric Gugelot
}

\section{RÉFÉRENCE}

Boniface Xavier, Histoire religieuse de la Grande Guerre, Paris, Fayard, 2014, 504 p.

Cabanel Patrick et Encrevé André (dir.), Les protestants français et la Première Guerre mondiale, Bulletin de la société d'histoire du Protestantisme français, Paris, SHPF-Droz, 2014, 545 p.

Moulinet Daniel, Prêtres soldats dans la Grande Guerre. Les clercs bourbonnais sous les drapeaux, Presses universitaires de Rennes, 2014, 331 p.

Nous les Poilus. Plus forte que l'acier. Lettres des tranchées à Thérèse de Lisieux, Paris, Éditions du Cerf, coll. «Épiphanie », 2014, 205 p.

1 Les commémorations se révèlent parfois fécondes pour la communauté scientifique. Ainsi la déferlante 1914-1918 concerne aussi les domaines des sciences sociales des religions. Au-delà du flot de conférences, expositions, la multiplication des publications livre autant d'ouvrages d'histoire que de témoignages, d'archives.

2 «La guerre est une expression de la civilisation tout autant et peut-être plus que de la barbarie... On fait la guerre pour un presque rien, mais qui est tout : une certaine manière de penser, de sentir... On fait la guerre pour une certaine façon de voir le monde. Toute guerre est une guerre de religion... Et en effet qui ne serait prêt à se faire tuer plutôt que d'accepter de voir désormais le bien et le mal, le beau et le laid, là où le voient nos ennemis $»{ }^{1}$.

3 Selon Jacques Rivière, alors prisonnier en Allemagne, cette guerre est une guerre de " civilisation » et donc une guerre de religion. $\mathrm{Au} \mathrm{xx}^{\mathrm{e}}$ siècle, la guerre mue en conflit total, ce qui en transforme profondément l'enjeu. 

1914-1918 est une étape ? Quel impact a le conflit sur la foi, les pratiques religieuses et les Églises?

(ande Guerre n'est pas une guerre de religion, mais un affrontement où les religions furent mobilisées par la guerre. Deux grands champs ont été particulièrement prospectés. Le premier est plutôt institutionnel et idéologique. Il cerne le rôle des Églises dans le conflit, avec notamment de nombreux travaux sur la prédication pendant la guerre ou le rôle du pape Benoît XV et sa note de paix en 1917. Le deuxième concerne l'étude des ferveurs et croyances des combattants et des civils ${ }^{2}$. La Grande Guerre n'est pas une guerre sainte menée au nom d'une idée religieuse ou d'un devoir divin. Pourtant le conflit, sa brutalité, sa durée, favorise l'émergence d'une « religion de guerre ». La guerre affecte la manière de croire et de vivre la foi. Les valeurs religieuses sont éprouvées à l'aune de l'angoisse, de la souffrance, de la blessure et de la mort de masse.

L'ouvrage de Xavier Boniface livre une synthèse de référence qui cerne l'ensemble des apports de l'historiographie, évoquant tous les belligérants et les religions.

7 Les Églises participent pleinement et volontairement au conflit. " Partout, les religions dans leur triple dimension, ecclésiale, doctrinale et croyante, se mobilisent en faveur de la guerre »(Boniface, p. 10). Elles incitent les fidèles à répondre aux mobilisations, bénissent les armées et légitiment les affrontements. Dès l'entrée en guerre, elles ne se distinguent pas des autres forces vives des sociétés européennes et le clergé s'associe au patriotisme ambiant. L'auteur replace justement le phénomène de " retours aux autels ». Ce moment de 1914 apparut comme porteur de renouveau spirituel. Le phénomène touche toutes les confessions mais s'essouffle rapidement à partir de 1915. Le livre de Daniel Moulinet témoigne de son impact chez de jeunes clercs. L'explosion de religiosité des premiers mois du conflit que l'on trouve tant chez les catholiques que chez les protestants, surprend par l'ampleur du phénomène, mais ces clercs découvrent rapidement que c'est un feu de paille : «Ce renouveau religieux dont on avait tant parlé au début des hostilités n'était guère que la crainte de la mort. Mais la foi n'est pas plus vive!» (Lettre de Monat, 23 juillet 1915, Moulinet, p. 112). La déception est vive : «Au début de la guerre, les églises étaient pleines de monde, les offices maintenant se célèbrent devant quelques soldats seulement. " (Lettre de Micard, 15 octobre 1917, Moulinet, p. 115) Les protestants ne sont pas épargnés : «Pendant les premiers mois de cette guerre, dans certaines de nos églises protestantes, on a constaté une ferveur visible, beaucoup plus grande. [...] ce que l'on vient aujourd'hui demander le plus souvent à la religion, je n'ai pas besoin de vous le dire [...]. Pour la plupart, vous êtes dans le deuil ou dans l'angoisse. [...] ce que l'on va chercher, dans les églises et dans les temples, c'est un peu de force pour supporter une croix que l'on est tenté de trouver trop lourde; ce que l'on veut, c'est revivifier les pauvres espérances que l'on porte en soi. »33.

8 L'influence religieuse reste diffuse et la mobilisation du clergé et des fidèles est réussie en 1914. L'effroi des pertes et l'horreur des combats, la durée de la guerre, et la lassitude qu'elle engendre ébranlent sans le remettre en cause l'engagement des sociétés. Les belligérants partagent le même sentiment de mener une guerre défensive, et donc une guerre juste. Cette vision favorise la nationalisation des religions d'autant que les grandes religions et confessions participent, comme les courants socialistes, de la paix civile et du consentement majoritaire au conflit. Les Églises légitiment une lutte menée pour la sauvegarde de la patrie et la défense des plus hautes valeurs spirituelles. Une prédication de combat se retrouve dans toutes les confessions. Les clercs insistent sur la mission 
singulière de la nation à laquelle ils appartiennent. Ainsi le pasteur du Havre, Jean Lafon affirme le 24 janvier 1915: "dans son unité matérielle et morale, elle [la France] représente certaine qualité d'âme qui lui confère une mission dans l'humanité. [...] la France, sûre de son droit et fidèle à sa tradition doit rester, au sein de l'humanité, comme l'incarnation des idées généreuses, comme la gardienne de ces principes abstraits dont la beauté éternelle l'emporte sur tous les calculs et tous les intérêts $»^{4}$. Néanmoins si les fluctuations de la pratique nourrissent peu les religions officielles, elles n'entravent pas un réel renforcement des communautés croyantes (Boniface, p. 120). Dans la lignée des travaux d'Annette Becker, l'ouvrage s'interroge sur la foi au quotidien dans une guerre mécanique et industrielle. La mort de masse bouleverse-t-elle les croyances? « Comment méconnaître dans la guerre les forces mystérieuses qui tantôt nous écrasent et tantôt nous sauvent. Je n'aurais jamais imaginé à quel point la guerre, même cette guerre moderne tout industrielle et savante, est pleine de religion $»^{5}$, écrit Robert Hertz en octobre 1914. La guerre meurtrit profondément les sociétés et fait "naître chez ces combattants de nouveaux systèmes de références et de représentations" (Boniface, p. 61). Si la guerre réveille parfois des sentiments anciens, les pratiques et expressions ne s'établissent pas nécessairement dans les religions établies. D'ailleurs l'impact religieux de la guerre est réel mais faible. En 1919, un curé de campagne jurassien note: «Les démobilisés rentrent. Pas plus pratiquants qu'avant la guerre.» (Boniface, p. 91). Néanmoins « une réaffirmation des croyances [...] et une réappropriation de traditions religieuses parfois délaissées » sont perceptibles (Boniface, p. 97).

La guerre devient l'horizon d'attente des populations, elle envahit les prières, les prêches, les mandements, les articles, les livres. L'ampleur des sacrifices suffit à justifier la poursuite de l'affrontement. L'appel à la paix blanche devenait alors impossible à entendre. La guerre représente néanmoins une faillite sur le plan de l'esprit. Messianismes politiques et religieux interprètent la guerre comme une cause sacrée de la civilisation et transforme le conflit en une mystique patriotique d'où peut sortir un monde nouveau et radieux, un monde purifié. C'est que foi et patrie se mêlent intimement. La paix civile règne partout et les débats religieux d'avant-guerre s'estompent. Même en France, l'anticléricalisme ne subsiste que sous forme résiduelle ou chez quelques adeptes d'une vision complotique. Rares sont les oppositions religieuses au conflit, quelques voix s'élèvent pour regretter que les belligérants mobilisent Dieu à leurs côtés. «Pour nous, prêtres-soldats [...], la guerre fut un baptême dans le Réel $»^{6}$, note le brancardier jésuite Pierre Teilhard de Chardin. Les aumôniers renouent avec les hommes, éprouvent le sentiment de «remasculiniser » la foi (Boniface, p. 89) : «Un aumônier qui va dans la tranchée donne naissance à un bataillon qui va à l'église » (Rév. Philip Clayton, p. 68).

10 Le numéro spécial du Bulletin de la société d'histoire du Protestantisme français, consacré aux protestants français et la Première Guerre mondiale, nous livre une réussite historiographique tant par le panel des vingt-deux articles que par le choix de les présenter en trois axes qui permet de cerner les protestants en guerre et dans la guerre. La première partie intitulée "Églises, presses, groupes et institutions", étudie l'adaptation des institutions de la nébuleuse protestante au contexte du conflit. La prédication est évoquée, les aumôniers protestants ne sont évidemment pas absents ainsi que diverses voix tels La Revue chrétienne et même le Bulletin de la société d'histoire du Protestantisme français. La seconde partie évoque les différentes « régions protestantes " (Bretagne, Charente-Poitou, Gironde-Dordogne, Cévennes, Ardèche-Drôme, Nord occupé). 
Elles font la commune expérience de la mobilisation, de la désorganisation des structures, des difficultés d'assurer avec des effectifs réduits toutes les charges, de faire face surtout à la multiplication des morts. Des particularités régionales ou confessionnelles (un article est consacré aux 2820 baptistes de France pendant la Grande Guerre) apparaissent. La troisième partie livre les portraits de quelques personnalités protestantes en guerre de l'oncle et du neveu, Charles et André Gide à Jules Puech ou Marguerite de WittSchlumberger, ou les plumes de Jean Norton-Cru ou d'André Chamson.

11 L'attitude des protestants pendant la guerre prouve qu'elle se différencie peu de celle de leurs compatriotes. Pourtant les relents de l'antiprotestantisme d'avant-guerre, qui les associaient à des semi-étrangers, pouvaient menacer « l'union sacrée ». S'ils refusent très tôt l'idée d'une guerre des religions, ni les institutions protestantes, ni les fidèles ne s'opposent à une guerre qu'ils pensent nécessaire et légitime face à l'agression allemande : «Le Conseil de la Fédération des Églises protestantes de France, au nom du protestantisme tout entier, exprime sa profonde douleur de voir, après tant de siècles de christianisme, deux grands empires violer systématiquement les règles les mieux établies du droit des gens; s'indigne avec toute l'humanité civilisée contre la destruction de Louvain et le bombardement de la cathédrale de Reims; réprouve l'abus des phrases pieuses dont les empereurs d'Allemagne et d'Autriche donnent le scandaleux exemple depuis le commencement des hostilités; constate avec tristesse combien cette exploitation de Dieu risque de compromettre la religion devant la conscience moderne [...] $»^{7}$. Or avant guerre, nombreux sont les Français à prêter un vif intérêt aux avancées exégétique et dogmatique du protestantisme allemand. En 1915, la Fédération protestante fonde le Comité protestant de propagande française à l'étranger, parallèle à celui des catholiques. La prédication est largement à charge. Elle insiste sur l'idée que l'avenir de l'humanité se joue dans le sort de la France: «d'autres nations ont leur personnalité légitime [...] mais notre mandat particulier est bien de propager, ici-bas, ces éternels principes de liberté, de justice, de fraternité, qui forment l'intangible Décalogue des temps modernes $»^{8}$. Cette vision messianique de la France se surimpose à un indéfectible attachement républicain. En face, ils dressent un portrait noir de l'Allemagne: "Quoi qu'ils puissent dire, nos agresseurs sont les représentants non certes pas d'un principe, mais de l'orgueil, de l'ambition, de la convoitise, de l'égoïsme, de la haine, par la suite, de l'esprit de conquête, de l'agression brutale, de la force, créant le droit de l'injustice, de la violence et du mépris de la vie humaine, en un mot, du mal dans toute son horreur $"$. Quant à la Revue chrétienne, qui ne parle plus que de la guerre, elle s'assigne pour tâche de défendre les positions françaises et indirectement les protestants français en réponse à des attaques de polémistes catholiques, mais n'engage aucune véritable réflexion théologique. (BHSPF, p.102) En contrepoint, un passionnant article évoque les prédications protestantes en Alsace pendant la guerre à l'occasion de l'anniversaire du kaiser. « Nous avons la certitude que la victoire de notre peuple est une victoire du bien au profit du monde. C'est l'Homme juste qui combat pour nous $»^{10}$. Ils affirment le lien étroit entre l'Allemagne et Dieu, entre le patriotisme et la foi. La guerre est une épreuve qui peut permettre de renouer avec les valeurs éternelles du peuple allemand (ibid., p. 64). Même si la métaphore du barbare semble rarement utilisée, les ennemis sont largement associés au monde des ténèbres et du mal.

12 L'absence des hommes conduit inévitablement à une forte féminisation des activités croyantes, surtout charitables et sanitaires (Boniface, p.142). Les femmes de pasteur doivent assurer pendant leur absence les tâches pastorales diverses. Très largement perçu 
comme une substitution, et un élargissement de leurs rôles traditionnels, cela peut aller jusqu'à des formes de "ministère conjoint» (BHSPF, p. 127) en particulier dans la prédication et les actes pastoraux (ibid., p. 129, 301, 348, 384 : un traitement est versé à l'épouse officialisant son activité) : «La mobilisation de mon mari m'a placée en face d'une lourde charge. [...] j'ai [...] aussi assuré la marche régulière de l'Église, école du dimanche, cours des catéchumènes, assuré l'ouverture du temple tous les dimanches, et quand le lecteur était absent, fait entièrement le culte. » (ibid., p. 129) La fin de la guerre marque néanmoins un retour aux rôles traditionnels. Ceci concerne d'ailleurs toutes les religions. Les femmes participent aussi d'un discours moralisateur qui appelle à la restauration des valeurs traditionnelles de la famille, de l'ordre et de l'autorité et qui insiste sur le refus de la jouissance et de l'anarchie. Leur mobilisation est très forte. Les dames d'œuvres, les groupes du catholicisme social féminin s'engagent dans le soin aux blessés, la correspondance et les colis pour les combattants.

Pour les différentes régions étudiées, une première distinction frappe, la différence entre territoires occupés et les autres. La présence allemande est peu tolérée. Le pasteur Kaltenbach à Saint-Quentin s'abstient de toute participation à une cérémonie officielle et d'accepter tout subside des forces occupantes (ibid., p. 261). Ainsi quand un inspecteur primaire allemand fait rajouter un crucifix dans une école de Ham, cela choque (ibid., p. 261). La vie spirituelle des communautés est contrariée par la guerre même si, à l'exemple de la Bretagne, l'arrivée de réfugiés peut brusquement augmenter l'auditoire des cultes du dimanche (ibid., p. 285). Les pasteurs manquent (ibid., p. 299). Les élections presbytérales sont largement suspendues pendant tout le conflit. En territoires occupés, à Roubaix et Tourcoing, le conseil presbytéral instaure des « cultes laïques » en août 1915 pris en charge par des fidèles un ou deux dimanches par mois pendant trois ans (ibid., p. 259).

14 L'expression de la foi reste peu étudiée. Ainsi l'impact de la guerre sur la vie spirituelle, en particulier la fréquentation du culte dominical, est difficile à cerner. Quelques notations néanmoins: "nos gens, au nom de la guerre, ont travaillé les dimanches comme les jours ordinaires et nos cultes ont bien souffert $»^{11}$. Ainsi un soldat confie à l'aumônier Bost qu'il n'a pas suivi le culte pendant " près d'un an ; aujourd'hui nous avons le bonheur d'en avoir un » (ibid., p. 111). Des différences sont néanmoins perceptibles. Les aumôniers protestants, semble-t-il, luttent plus que leurs homologues catholiques contre l'abus d'alcool (ibid., p. 114, à l'exception des régions de commerce du cognac et du pineau, ibid., p. 307). Il semble aussi que sur les pratiques autour de la mort, des emprunts soient faits auprès des catholiques face à une demande des soldats (ibid., p. 115). Les aumôniers protestants, comme les aumôniers catholiques et juifs, sont contraints à un œcuménisme réel même s'il est limité. En décembre 1915, à quelques jours d'intervalle, le pasteur Durrleman officie avec un prêtre catholique lors d'obsèques et lit des psaumes pour l'enterrement d'un jeune juif (ibid., p. 119).

L'histoire du BHSPF montre l'engagement des protestants y compris dans la lutte des idées. La revue répond au "Manifeste des 93 » intellectuels allemands qui rejette l'idée d'une responsabilité allemande dans le déclenchement et la sauvagerie du conflit. Il se mobilise en faveur d'une "guerre culturelle» (BHSPF, p. 236), et célèbre les grands anniversaires de la Réforme, 1915 bicentenaire du premier synode du Désert et 1917 quatrième centenaire de la Réformation. Même si Alfred Cadier écrit dans l'éditorial de $L a$ Fraternité le 7 décembre 1918, "C'est Calvin qui a battu Luther»(ibid., p. 317), les positions protestantes distinguent largement le réformateur de son pays d'origine : « $\mathrm{Si}$ 
nous célébrons Wittenberg, c'est en nous refusant, de la manière la plus expresse, à unir religieusement l'Allemagne de Luther à l'Allemagne d'aujourd'hui sur laquelle pèse la responsabilité de la plus odieuse et de la plus criminelle des guerres qui aient jamais désolé le monde. $»^{12}$ Néanmoins les luthériens restent les grands absents du Bulletin. « La guerre a [...] confirmé que le droit valait plus que la foi, la Nation davantage que la confession » (ibid., p. 240).

$\mathrm{Au}$ sein du protestantisme aussi, les rares oppositions à la guerre ne s'expriment pleinement qu'après le conflit. Quant à la dissidence tout individuelle du héros de la fiction de Chamson, Roux, elle reflète l'exigence des valeurs évangéliques au milieu des années 1920 dans un contexte de large pacifisme ambiant. Même Jules Puech socialisant et pacifiste avant 1914, s'engage bien que réformé parce que seule une victoire contre le militarisme allemand permet à ses yeux d'instaurer une paix définitive.

La piété semble plus visible (ibid., p. 304) mais le doute existe aussi : «Oh ces mois, ces journées terribles nous rapprochent du Dieu d'amour en toute humilité et fidélité. Je ne comprends pas, mais quand je vois tant d'amis chrétiens frappés, quand je vois les crimes commis par des peuples chrétiens, je ne comprends pas, mais je veux croire malgré tout à la bonté de Dieu. ${ }^{13}$ Car ce qui étreint tous ces croyants et une identique interrogation: comment croire au cœur d'un tel massacre? « Pourquoi Dieu n'arrête-t-il pas la guerre? Tantôt ce sont des incrédules qui nous lancent la question ironiquement à la tête, comme une objection décisive à notre foi chrétienne, tantôt ce sont des croyants qui la murmurent avec angoisse, se demandant pourquoi Dieu paraît sourd à leurs supplications, insensible à leurs souffrances. $\gg^{14}$ En Bretagne, l'auteur note que si les pasteurs perçoivent un moment d'approfondissement de la piété face aux épreuves, le mouvement de sécularisation se poursuit ainsi que «la montée du matérialisme » (ibid., p. 288). Le doute, l'angoisse, l'épreuve mine néanmoins. Certains y approfondissent leur foi, d'autres s'éloignent.

pêtres et séminaristes bourbonnais écrivent, durant tout le conflit, à l'abbé Giraud, leur supérieur du séminaire de Moulins, un diocèse en voie de détachement mais qui comprend encore un clergé abondant. Ces lettres assurent un lien vital entre tous ces clercs. "Ces lettres sont si bonnes et ce "mot de Jésus" que vous aimez à nous dire que nous attendons chaque fois, est pour nous si plein de grâce : c'est un renfort de munitions contre les ennemis de nos âmes. » (Busserolles, 3 juin 1915, Moulinet, p. 187).

L'auteur rappelle combien ces clercs constituent un sous-groupe particulier. Ils sont environ 200 à partir, 20 n'en reviendront pas. 25000 prêtres, séminaristes et religieux catholiques sont mobilisés en 1914. Ils sont 32699 durant le conflit, dont 23418 séculiers et 9281 réguliers. La moitié est affectée au service de santé et l'autre parmi les combattants. Bien qu'étroit, ce panel permet de cerner la façon dont ces clercs vivent certains moments de la guerre, dont ils accommodent leurs devoirs religieux et militaires, comment ils développent une vision croyante du conflit et une spiritualité de guerre alors même que leur culture et leur formation en font des hommes volontairement séparés de leurs contemporains.

Comment en effet conserver une vie de l'âme et de l'esprit dans les conditions de la guerre moderne? " C'est une vie tellement abrutissante, la vie au front, il n'y a guère que la bête qui vive.» (Lettre de l'abbé Vérillaud, 24 juin 1915, Moulinet, p. 137) Toute vie intérieure est bien difficile : «J'en arrive à ce point que je pense à Jésus juste en me levant et avant de me mettre au lit !» (Lettre d'Ernest Aurambout, 11 juin 1915, Moulinet, p. 43) Elle est soutenue par quelques lectures, surtout L'Imitation (Moulinet, p. 195-196), L'âme de 
tout apostolat de Dom Chautard (Moulinet, p. 197), mais aussi Psichari. Ces prêtres soldats ne parviennent pas à respecter les conditions de la célébration des messes, de la lecture du bréviaire, du jeune eucharistique. La prière et le chapelet deviennent leurs refuges. «La vie des tranchées est abrutissante et l'on perd facilement le goût du surnaturel » (Moulinet, Monat, 28 juin 1915, p. 231). Le Sacré-Cœur, la Vierge et Jeanne d'Arc composent un panthéon de référence pour leurs dévotions personnelles (Moulinet, p. 259-266).

21 La haine de l'ennemi est réelle : «Autour de moi, je n'entends bien souvent que des cris de haine contre les ennemis qui nous font la guerre. Tous les cœurs ne respirent que vengeance " (Lettre de Micard, 18 février 1915, Moulinet, p. 154). Une foi commune ne suffit pas : «Ces gens-là ne méritent plus aucune pitié, car ils ont tout fait, même insulté Dieu en le parodiant à leur façon, Unser Gott. Ils se disent religieux et pour cela portent crânement sur leurs casques leur célèbre devise: Gott mit uns für König und Vaterland. Cependant leur première besogne, partout où ils passent est de s'acharner contre des monuments religieux et de méconnaitre les plus élémentaires principes de la loi divine. Que de pauvres églises j'ai vues victimes de leur barbarie et qui pourtant n'offraient absolument rien au point de vue stratégique !» (Lettre de Tronel, 11 avril 1915, Moulinet, p.155) Que l'Allemagne soit majoritairement protestante approfondit une lecture religieuse du conflit, mais c'est aussi une difficulté pour les Églises réformées. Dans Le Huguenot du 15 novembre et $1^{\mathrm{er}}$ décembre 1914, l'éditorial de V.Poux publie intégralement l'article du pasteur Guillaume Granier paru dans Le Christianisme au XX siècle où il dénonce les «vandales spirituels » c'est-à-dire la théologie allemande, la "science germanique » qui a développé cette exégèse longtemps admirée en France qui " sous d'honnêtes dehors pieux » a "infiltré son venin dans les esprits, et on peut, sans courir le risque de se tromper, la déclarer, pour la plus large part, responsable des misères humiliantes, schisme, scepticisme, incrédulité dont souffre notre monde protestant. » (BHSPF, p. 360-361) Une mise à distance envers l'Allemagne se dessine par exemple dans le mensuel, $L^{\prime} E c h o$ de la Drôme d'octobre 1914, qui publie un texte du pasteur Henry Dartigue où il dénonce la barbarie allemande lors de l'invasion : « Nous déclarons bien haut que nous ne pouvons plus tendre une main fraternelle aux Églises protestantes d'Allemagne, jusqu'à ce qu'elles aient confessé résolument, devant Dieu et devant l'Europe, dans la sincérité du repentir, les crimes de leur peuple et de leur empereur. » ( BHSPF, p. 380)

La durée du conflit transforme la croisade en chemin de croix. Les thèmes de la douleur et du deuil sont de plus en plus présents. Les sacrifices, les blessures et les morts s'assimilent à une " cène »: «Le vin du Christ est le sang allemand » (Walter Flex, Boniface, p. 250). L'insistance portée sur le devoir chrétien du sacrifice, sur la valeur régénératrice de la souffrance permet aux soldats chrétiens d'assimiler leur sacrifice à la passion du Christ qui sauve l'humanité: "Nous sommes actuellement jetés dans la fournaise de la souffrance. Il y en a pour tous; il y en a de tout genre. Rares sont ceux qui ont été épargnés ou le seront. Regardons la réalité en face: la vie a donné à chacun ce mot d'ordre: "souffrir" [...] Souffrir! Souffrir encore et sans cesse $! »^{15}$ Une certaine vision tragique de la foi chrétienne l'emporte sur l'idée de prêcher un Dieu amour. La propagande de guerre est envahie d'imagerie religieuse qui fait de la guerre un lieu de rédemption. «Les valeurs religieuses et guerrières tentent à se confondre dans les figures du héros, du saint et du martyr, adaptées aux circonstances de la guerre » (Boniface, p. 251). Chez les catholiques, la lecture religieuse du conflit l'interprète comme une 
punition pour les péchés de la France mais aussi comme salvatrice : « Notre pauvre pays a bien péché et il expie ses fautes d'une façon terrible qui devrait ouvrir bien des yeux. » (Micard, 9 décembre 1917, Moulinet, p. 163) Une lecture théologique du conflit permet d'interpréter le bain de sang: "On ne se souvient pas qu'il faut un sang pur pour laver la souillure du péché. Quelle admirable consolation que la religion chrétienne en ces temps de guerre, et combien les événements actuels se trouvent expliqués par le dogme éminemment catholique de la communion des saints ou de la réversibilité des mérites si bien décrits au siècle dernier par de grands catholiques tels que de Maistre, Coppée, Huysmans... » (Vichy, 7 mars 1915, Moulinet, p. 160). Consolation, le mot résonne comme une autre vision des religions en guerre. La défiance envers la République se maintient malgré la paix civile : « tant de sang versé, tant de sacrifices consentis, tant de larmes des mères, des veuves et des orphelins nous devraient mériter d'avoir une France plus belle... mais les meilleurs des enfants se font tuer, les canailles et les bandits restent loin du danger et des armées et pendant que les autres servent la patrie, eux la trahissent et se préparent à être les maîtres de l'avenir par la propagande la plus éhontée " (Lettre de G. Malvielle à ses parents, 18 août 1915, Moulinet, p. 133). Néanmoins « une œcuménicité de fait » (E. Fouilloux) apparait : « Il n'y a pas d'église, la messe a été célébrée ce matin à l'école laïque, la voilà l'union sacrée » (Lettre d'Ernest Aurambout, 5 mars 1916, Moulinet, p. 43). Les rares occasions de proximité entre catholiques et protestants sont dues aux conditions du conflit. Fernand Carrel, aumônier au $119^{\mathrm{e}}$ Régiment d'Infanterie, rapporte la découverte d'un sommier et d'un matelas avec deux aumôniers d'autres confessions et l'officier: "Vite on tire au sort, le pasteur couche avec le rabbin (l'Ancien avec le Nouveau Testament) et le dogme que je représente s'allonge aux côtés de la libre pensée » ${ }^{16}$. Les cérémonies patriotiques en sont souvent l'occasion (" prière nationale », BHSPF, p. 382) pendant la guerre mais aussi après lors de l'érection des monuments aux morts ( ibid., p. 314). Ainsi le pasteur John-David Bost accepte lors de la célébration du «Souvenir français ", l'invitation à une cérémonie dans la cathédrale de Rennes et "d'associer les prières de son cœur et celles de l'Église Romaine, dans la large mesure où le permet une foi commune en Dieu, en Jésus-Christ et en la vie éternelle, ainsi que l'amour commun pour la patrie terrestre» (ibid., p. 287). Des dissidences apparaissent néanmoins. La plupart des temples inaugure des plaques commémoratives pour les fidèles morts pendant la guerre (ibid., p. 315, 329, 391-393) mais à Bordeaux, le 2 novembre 1920, le conseil presbytéral refuse l'accès au temple pour la cérémonie organisée aussi à la cathédrale et à la synagogue par la Société des combattants " au motif qu'il ne convient pas de prier pour les morts » (ibid., p. 329). Même la «fraternité des tranchées » connaît des limites du côté des clercs (Moulinet, p. 130): "J'offre toutes mes peines à Notre Seigneur, surtout quand je suis obligé de vivre avec les hommes de mon escouade, gens sans éducation, sans morale et ne sachant que mal parler de la religion. » (I. Lafaye, 3 août 1917, Moulinet, p. 234) Ces quelques remarques modèrent l'idée communément partagée d'un chaudron des tranchées.

La foi se heurte à l'horreur du conflit : «Cette guerre sera un mal pour tout le monde. C'est une école malsaine, où l'on prendra l'habitude des gestes criminels, des désirs de vengeance, des pensées absolument contraire à l'esprit de l'Évangile. [...] on rit d'entendre des hurlements de douleur dans les tranchées d'en face et quand un de nos tirs de barrage [...] fait voler aux quatre coins de l'horizon les bras, les têtes et les jambes. [...] Nous sommes les attaqués. Les Allemands se font tuer chez nous par milliers, mais ils ne veulent pas partir. Nous sommes obligés de les démolir. Et voilà justement ce qui est terrible pour nous: nous faisons notre devoir en tuant. $»^{17}$ Témoignages catholiques et 
protestants prouvent combien la violence de guerre heurte ces croyants sans jamais les entraîner dans la dissidence. Ainsi, les prêtres participent d'une œuvre de destruction, de mort, loin des attendus de leur engagement spirituel: "tout est organisé pour la destruction et l'on ne travaille que pour cela: même nous, guérir pour détruire.» (Moulinet, 4 mars 1915, p. 48) Ils savent que leur devoir de soldat entre en contradiction avec le décalogue. "J'ai besoin plus que jamais de vos prières et de celles de mes frères car bientôt je vais aller tuer.» (Moulinet, 28 février 1916, p. 98) Ces soldats chrétiens, qu'ils fussent prêtres ou non, apprennent à tuer, tuent: "Me voilà encore à Bourges, instruisant toujours les bleus, leur apprenant les moyens de tuer sans se faire tuer. C'est un peu barbare, je l'avoue, surtout pour un séminariste qui, si Dieu le permet, doit prêcher un jour la paix, la douceur, la bonté! Je tâche de me dédouaner en priant de temps en temps pour les soldats de toutes les nations. " (Moulinet, lettre d'Aurambout, 13 novembre 1914, p. 232) Deux témoignages montrent cette déchirure. Le premier est protestant : «Oh ! je les entendais, ils étaient une vingtaine, des pères de famille peut-être comme moi, et j'avais mes grenades en main et je me disais ; je vais faire des morts, des veuves et des orphelins; et j'ai jeté cependant et j'ai entendu les cris, j'avais froid au cœur, je tremblais de tout mon corps. $\aleph^{18}$ (BHSPF, p. 100) Car le croyant reste un combattant comme en témoigne cet artilleur catholique : «En observant un tir de 155, il pense que ces obus vont tuer " $5,10,15$ malheureux, 15 hommes. Et du même coup avant qu'il ne tombe, je prie pour leur âme; et en même temps, je me surprends à dire: Mon Dieu faites que le coup porte" ${ }^{19}$. De petits interstices de pacification dans un monde de violence surgissent même quand aux détours des lettres, on découvre qu'un prêtre fut désigné nettoyeur de tranchées : «Parti comme nettoyeur de tranchées, je n'ai pas eu à me servir de mes armes. Tous les Allemands que je rencontrais se sont rendus ou étaient déjà mortellement atteints. Non seulement je n'ai pas eu à faire encore de mort ; mais j'ai même pu empêcher un jeune soldat de tirer sur un prisonnier. Que Dieu est bon de m'avoir épargné le cruel devoir de verser le sang. Il conduit aux enfers et en ramène. » (Lettre de Grach, 9 novembre 1917, Moulinet, p. 233) Le nettoyeur de tranchées a pour fonction d'éliminer les captifs pris lors d'un assaut quand ils deviennent encombrants ou une menace pour les attaquants. Le trouble existe néanmoins et déchire les consciences : « Ennemis !... Aimez-vous les uns les autres, a dit le Christ... Et moi, de mon côté, je règle sur eux la trajectoire mortelle... Bêtise ! Infamie !... Un dégoût m'envahit : une immense nausée de cette guerre ignoble, de ce sang, de cette fange, de cette putréfaction. $»^{20}$

Les soldats et leurs familles ont cherché dans leur habitus religieux une consolation face à la mort de masse grâce à l'intercession de multiples protecteurs, le Sacré-Cœur (un million et demi de représentations distribuées pendant la guerre), les saints protecteurs de la France auxquels s'ajoutent la bienheureuse Jeanne d'Arc et Thérèse de l'Enfant Jésus. Les multiples ex-voto des lieux de pèlerinages montrent la reconnaissance pour la protection obtenue.

Le livre consacré aux lettres de tranchées à Thérèse de Lisieux, au demeurant largement absente des lettres des prêtres du Bourbonnais (Moulinet, p. 266), est un recueil, une sélection de lettres reçues par le sanctuaire de Lisieux pendant le conflit. Il ne comporte aucun appareil critique ni bibliographique. Mais il nous livre des témoignages de cette ferveur et de cette foi qui put s'exprimer et se vivre dans l'enfer des tranchées. Le déluge de fer et d'acier a en effet engendré un déluge de lettres, parfois une centaine par jour au sanctuaire de Lisieux. 75 d'entre elles témoignent du rôle d'intercesseur de la " petite sœur » : «Cette sainte [ce qu'elle n'est pas encore] m'attire parce qu'elle me semble être 
l'incarnation de la bonté, de la simplicité, de la confiance filiale envers Dieu : il me semble qu'elle doit bien aimer les âmes qui recourent à elle. » (20 juillet 1916, Thérèse, p. 90)

La lecture de ces courriers montre que le culte de Thérèse de Lisieux se diffuse par l'intermédiaire de parents, d'amis, de camarades et de quelques clercs. La circulation de livres surtout de l'Histoire de ma vie, de reliques, de médailles et surtout d'images joue un rôle particulier dans son enracinement. «Je suis parti [...] sur le front, en emportant sur moi un portrait et une relique de la "petite sainte", comme des gages de préservation et d'heureux retour au foyer. » (30 avril 1916, Thérèse, p. 73) Famille et combattants croient au rôle protecteur de la carmélite, "la si douce Protectrice de ceux qui souffrent " (31 décembre 1916, Thérèse, p. 113). Elle apparait surtout comme une protectrice particulière des soldats: «La pluie de roses de la petite sœur Thérèse est le plus invulnérable des boucliers contre la mitraille boche et tous les autres dangers de cette vie. " (21 mai 1917, Thérèse, p. 128) Ces soldats témoignent de nombreux signes de protection obtenus par l'appel à la religieuse, nombreuses apparitions, odeur persistante (rose et violette) : « La bataille faisait rage, je la vis debout, au pied d'une mitrailleuse qui était là. Elle me regardait et nous bénissait tous. » (14 septembre 1916, Thérèse, p. 96) Ces lettres montrent l'enracinement de ce culte par les lettres, les demandes de messe et les pèlerinages de remerciements à Lisieux.

La revue Études a eu l'excellente idée de revenir sur sa longue histoire à l'aune du thème qui ici nous intéresse. Elle publie un hors-série qui regroupe un certain nombre d'articles, publiés depuis 1914 sur la guerre. Une première partie se rapporte à 1914-1918 avec des textes de témoignages et de réflexion de jésuites tels L. de Grandmaison, Y. La Brière, L. Roure, J. Lebreton... et le célèbre texte de Teilhard de Chardin, lui-même au front, et les suites dans l'entre-deux-guerres (G. Fessard, P. Doncœur). Une deuxième partie se rapporte à l'après 1945 (J.Sommet, P. Teilhard, Y.Calvez, P. Valadier). L'importance même des signatures donne tout l'intérêt de ce numéro qui nous livre ainsi des documents passionnants sur la réflexion catholique autour de la guerre et de la paix sur la durée d'un siècle de conflits.

L'impact du conflit n'est pas négligeable. "L'appel aux forces et aux influences morales aura été l'un des caractères, non pas inédits, mais très remarquables de la grande guerre du vingtième siècle. La guerre de plume, la guerre d'idées, s'est juxtaposée [...] aux opérations des armées [...]. Chaque belligérant a voulu mobiliser les forces spirituelles, les puissances morales, pour entretenir chez ses propres nationaux les énergies guerrières et les espérances de victoire [...]. Les forces morales et spirituelles ont été encore mises en action [...] pour l'exercice continuel de la fraternité patriotique et de charité chrétienne. [...]. La plus haute puissance morale qui eut un rôle dans la guerre fut la force religieuse. » ${ }^{21}$ La Grande Guerre n'est pas sans foi. Celle-ci joue un rôle crucial, foi en la patrie, foi en la victoire relayée par la foi en Dieu ou par d'autres croyances. Les Églises prennent rang dans la mobilisation générale et soutiennent l'effort de guerre. Le soldat et sa famille recherchent de multiples assurances, celle de l'affection de sa famille, celle de la patrie et celle de la croyance. Si la guerre a favorisé la résurgence d'une religiosité enracinée dans l'expérience humaine, elle a aussi impulsé des innovations spirituelles (le culte de Thérèse) et marqué surtout la lente disparition des conflits religieux d'avant 1914. Reste le poids du deuil et ce soulagement terrible de la fin du conflit : « Le sang ne coule plus. Nous pouvons nous étendre le soir dans nos lits sans nous dire : cette nuit il y aura des morts, des blessés, hurlant de douleur dans l'ombre. [...]. C'est fini. Le canon ne tonne plus [...] le cœur des mères ne bat plus à chaque courrier. $»^{22}$ 


\section{NOTES}

1. Jacques Rivière, À la trace de Dieu, Gallimard, coll. «Blanche", 1925, p. 37. Cité par Annette Becker, La guerre et la foi. De la mort à la mémoire 1914-1930, A. Colin, coll. «U Histoire contemporaine ", 1994, p. 15.

2. L'historiographie de la Grande Guerre disposait déjà de nombreuses références. En voici quelques-unes non citées dans la note :

- Des hommes d'Église dans la Grande Guerre, Guerres mondiales et conflits contemporains, ${ }^{\circ}{ }^{\circ} 187$, juillet 1997.

- Pour une histoire religieuse de la guerre, 14-18 Aujourd'hui, Today, Heute, $\mathrm{n}^{\circ} 1$, Noésis, février 1998.

- Xavier Boniface, L'Aumônerie française 1914-1962. Paris, Éditions du Cerf, coll. « Histoire religieuse de la France », 2001.

- Jacques Fontana, Les catholiques français pendant la Grande Guerre, Éditions du Cerf, coll. «Cerf Histoire ", 1990.

- Étienne Fouilloux, "Première guerre mondiale et changement religieux en Europe ", dans Jean-Jacques Becker et Stéphane Audoin-Rouzeau (dir.), Les sociétés européennes et la guerre de 1914-1918, Université de Paris X, 1990.

- Philippe Landau, Les Juifs de France et la Grande Guerre. Un patriotisme républicain, CNRS Éditions, 1999.

- Jean-Marie Mayeur, « La vie religieuse en France pendant la Première Guerre mondiale ", dans Histoire vécue du peuple chrétien, Privat, 1979.

3. Raoul Allier, Allons-nous vers une Renaissance religieuse?, Conférence prononcée dans le Temple de l'Oratoire, le 15 juin 1915, Paris, Librairie de Foi et Vie, 1915, p. 6-8.

4. Jean Lafon, «L'héritage des Pères ", 24 janvier 1915, p. 56. Cité dans Laurent Gambarotto, Foi et patrie. La prédication du protestantisme français pendant la Première Guerre mondiale, Labor et Fides, coll. « Histoire », 1996, p. 139.

5. Lettre de Robert Hertz du 3 octobre 1914, Un ethnologue dans les tranchées. Lettres de Robert Hertz à sa femme Alice, Paris, CNRS Editions, 2002, p. 69-70.

6. Pierre Teilhard de Chardin, Genèse d'une pensée. Lettres 1914-1919, Paris, Grasset, 1961, p. 37.

7. Déclaration du Conseil de la Fédération des Églises protestantes de France, 28 sept. 1914, cité dans Le Christianisme au XX ${ }^{e}$ siècle, $1^{\mathrm{er}}-8$ oct. 1914, p. 325.

8. Wilfred Monod, « Le Nom de l'Eternel », Paris, 23 août 1914, dans Gambaretto, BHSPF, p. 39.

9. Louis Trial, « Motifs d'espérance », Nîmes, 30 août 1914 dans Gambaretto, SHPF, p. 40.

10. Johannes Ficker, 27 janvier 1917, cité p. 67.

11. Bulletin évangélique. Journal des Églises réformées de l'Ouest, juin 1917 (BHSPF, p. 303).

12. Franck Puaux, «Discours de M. le Président », BHSPF, 1917/3, p. 274. Cité dans BHSPF, 2014, p. 237.

13. Journal de Louise Dentan, $1^{\text {er }}$ février 1916, p. 351.

14. Journal du Pasteur Kaltenbach, 6 août 1916, cité dans BHSPF, p. 264.

15. H. Gambier, «Une offre de Jésus-Christ », 11 février 1917, p. 1. Cité par Gambarotto, Op. cit., p. 71-72.

16. Nadine-Josette Chaline, "Les aumôniers catholiques ", dans Nadine-Josette Chaline (dir.), Chrétiens dans la Première Guerre mondiale, Paris, Éditions du Cerf, coll. « Histoire religieuse de la France ", 1993, p. 107. 
17. Lettre d'un prêtre combattant, Semaines religieuses de Bayeux, 19 août 1917. Cité par Dominique-Marie Dauzet, «Entre patriotisme et réalisme », dans Séverine Blenner et Jacqueline Lalouette (dir.), Servir Dieu en temps de guerre. Guerre et clergés à l'époque contemporaine (XIXe-XXI siècles), Paris, A. Colin, coll. « Recherches », 2013, p. 172.

18. Témoignage d'un soldat, cité par Charles Ducasse, «Le Nouveau Testament, la Guerre et le Chrétien ", Revue Chrétienne, 1916, p. 254.

19. Témoignage de l'artilleur L. B., Études, 5 mai 1915.

20. Témoignage du franciscain belge Martial Lekeux, Mes cloîtres dans la tempête, Paris, Plon, 1922, p. 11.

21. Yves La Brière, «Le rôle des forces morales dans la Grande Guerre », Études, 5 mars 1919, p. 614-620.

22. Le pasteur luthérien John Viénot, Revue chrétienne, décembre 1918. BHSPF, p. 339-415.

\section{AUTEURS}

\section{FRÉDÉRIC GUGELOT}

Université de Reims, frederic.gugelot@univ-reims.fr 